\title{
Calidad de vida, sentido de coherencia y satisfacción laboral en profesores(as) de colegios técnicos en la Dirección Regional de Heredia
}

\author{
Pedro Ureña Bonilla ${ }^{1}$ \\ Profesor en la Escuela de Ciencias del Deporte de la Universidad Nacional \\ Heredia, Costa Rica \\ Carmen Castro Sancho ${ }^{2}$ \\ Directora del Colegio Técnico Profesional de Flores \\ Heredia, Costa Rica
}

Recibido 2 de setiembre de 2008 • Aprobado 23 de marzo de 2009

\begin{abstract}
Resumen. El propósito del estudio fue analizar la calidad de vida, el sentido de coherencia y la satisfacción laboral en docentes de colegios técnicos de la región de Heredia. Participó un grupo de 81 educadores(as): 25 hombres y 56 mujeres. Se aplicó el cuestionario SF-36, las escalas de Sentido de Coherencia y de Satisfacción laboral. Los resultados encontrados mostraron la existencia de tres factores importantes relacionados con el bienestar laboral de los(as) educadores(as): el primero de ellos vinculado con el ambiente social y el reconocimiento del trabajo, el segundo asociado a las condiciones infraestructurales, remuneración y de ascenso y el tercero, caracterizado por el tipo y cantidad de trabajo y la relación con los estudiantes. Especialmente crítica resultó la satisfacción laboral de los(as) encuestados(as) en relación con las condiciones de infraestructura, posibilidades de ascenso y de remuneración. La calidad de vida es, en términos generales, aceptable; sin embargo en aspectos centrales como la vitalidad, se evidenció un deterioro importante. Los aspectos mejor calificados se refirieron a la función física y al rol físico. El sentido de coherencia mostró calificaciones aceptables, tanto para el factor vida como para el factor sentimientos. Ambos se relacionaron de manera significativa con dimensiones centrales de la calidad de vida y del bienestar laboral. En general, los resultados encontrados hacen evidente la necesidad de desarrollar e implementar programas que fortalezcan el sentido de coherencia y la calidad de vida de los(as) educadores(as); asimismo, dejan entrever la importancia de atender el tema de la satisfacción laboral, especialmente teniendo claro que,
\end{abstract}

\footnotetext{
Doctor en Ciencias del Deporte con especialidad en Psicología Deportiva, por la Universidad de Deportes, Colonia, Alemania. Ha sido decano y vicedecano de la Facultad de Ciencias de la Salud; así como también director interino de la Escuela Ciencias del Deporte de la Universidad Nacional y del Programa de Maestría en Salud Integral y Movimiento Humano. Correo electrónico: purena 21@ hotmail.com

2 Máster en Administración Educativa. Directora del Colegio Técnico Profesional de Flores. Heredia. Ha impartido cursos en el nivel universitario en áreas propias de la Administración y la Planificación educativa. Correo electrónico: carmencastros@hotmail.com
} 
una buena satisfacción laboral no sólo tiene consecuencias positivas sobre la salud, sino también sobre los niveles de compromiso e identificación del(la) educador(a) con el ejercicio de la profesión.

Palabras clave. Calidad de vida, sentido de coherencia, satisfacción laboral, salud.

Abstract. The aim of this study was to analyze the Quality of Life, Sense of Coherence and Job Satisfaction by teachers of technical schools in the Herediás region. A group of 81 educators (25 male and 56 female) joined the study. It was used the SF-36 Questionnaire, the Sense of Coherence scale and a list of items related to the Job Satisfaction. There were found three factors associated to job satisfaction: (1) social atmosphere and job recognition, (2) infrastructure conditions, salary and promotion and (3) amount of job and relationship with the students. In general the results showed scores up of average Quality of Life. However, there were found low scores in central aspects like "Vitality". The highest factors scores were found by the physical function and the physical role. The sense of coherence scores related to the factors "Life" and "Feelings" were middle. Both factors were significant associated to some dimensions of Quality of Life and Job Satisfaction. In sum, the results showed up, that it is necessary to develop programs in order to enhance sense of coherence and quality of life. Likewise, it is important to promote job satisfaction. A good job satisfaction has positive consequences to the health, and increase the compromise levels and teachers identification to the profession.

Key words. Quality of life, sense of coherence, job satisfaction, health.

\section{REFERENTE TEÓRICO}

En el marco de lo que podría denominarse Comunidades educativas saludables, el tema calidad de vida y satisfacción laboral están íntimamente relacionados y su atención es central en la generación de atmósferas de trabajo positivas, así como en el buen desempeño docente.

El concepto calidad de vida es multidimensional (Simón, 1999), y está ligado a constructos como felicidad y bienestar (Nordenfeld, 1993; Diener, 1994). Sin embargo, no se cuenta aún con una definición consensuada. Por ejemplo, Levi y Anderson (1980) entienden la calidad de vida como un concepto formado por las dimensiones bienestar físico, mental y social; aunque el nivel de bienestar está determinado por la valoración subjetiva que haga la persona. La felicidad, la satisfacción y la recompensa, son consecuencias del nivel de bienestar presente. De manera que, todo lo que produzca esta sensación incide, positivamente, en la calidad de vida (Diener). En sentido similar, Brengelmann (1986) asocia la calidad de vida a situaciones específicas, entre las que destaca: el disfrute de libertad, el cultivo de relaciones sociales, la posibilidad de desarrollar la propia iniciativa, la satisfacción, la ausencia de problemas psicosomáticos, el tener una buena profesión, un buen empleo y, finalmente, encontrarle sentido a la vida. En consecuencia, si se cumple una o varias de las condiciones mencionadas, y, además, se juzgan positivamente, se tendría como resultado lo que Veenhoven (1991) denomina un nivel de "satisfacción vital" favorable, y una mejor calidad de vida.

Como complemento a las propuestas anteriores, Simón (1999) afirma que cualquier definición de calidad de vida debe considerar tanto las capacidades cognoscitivas como las conductuales y el bienestar emocional. Asimismo, debe incorporar el sentimiento subjetivo de bienestar y la satisfacción vital, y paralelo a ello, las condiciones materiales objetivas de existencia y la percepción subjetiva de ellas. Por otro lado, es preciso incorporar la satisfacción derivada de las condiciones materiales reales y concretas, así como psicológicas de las personas. De manera paralela, debe valorarse la percepción subjetiva del nivel de salud personal y su capacidad para comportarse de una forma subjetivamente satisfactoria. Por último, debe incorporar la percepción de la utilidad del significado de vivir y la satisfacción de las necesidades básicas del ser humano. 
La satisfacción y el bienestar son aspectos centrales en el contexto de una buena calidad de vida y son el resultado de la percepción del logro de esperanzas y aspiraciones individuales (Duro, 2005). De manera que, ambos conceptos llevan implícita la vivencia personal subjetiva. De conformidad con esta línea de pensamiento, Ryff (1989) identifica seis áreas básicas para determinar el bienestar de las personas, ellas son: la autonomía, la auto-aceptación, el crecimiento personal, el dominio (control), las metas en la vida y las relaciones positivas. En consecuencia, cualquier contexto laboral que tome en cuenta estas áreas para estructurar la dinámica de las interacciones alrededor de fines y propósitos compartidos, estará promoviendo la sensación de bienestar, la calidad de vida del trabajador y la salud, en general (Santos, 2000, Requena, 2000; Bravo, Peiró y Rodríguez, 1996). El bienestar constituye así, un indicador positivo de la relación de la persona consigo misma y con su contexto laboral, e incluye aspectos valorativos y afectivos que se integran en la proyección presente, futura y en el sentido de vida del trabajador (Ryff, 1989). El bienestar laboral es, en consecuencia, un factor determinante de la calidad de vida de los(as) trabajadores(as) de cualquier institución.

Sin lugar a dudas, la calidad de vida está íntimamente relacionada con la salud (Sánchez, 1996). De manera que, al promocionar una mejor calidad de vida, se está promoviendo una mejor salud. En este contexto, se entiende la salud, como un proceso de relaciones dinámicas y bidireccionales entre dimensiones y competencias individuales (biopsicosociales) y características ambientales (biofísicas, sanitarias, socioeconómicas y culturales) cuyo resultado es un estado caracterizado por el equilibrio y el correspondiente bienestar psicosocial (Godoy, 1999).

Con este marco de referencia y centrando la atención en la dimensión de las competencias psicológicas, el Sentido de coherencia (Antonowsky, 1987) puede entenderse como un factor potenciador de la salud y, en consecuencia, de la calidad de vida (Ebert, Tucker y Roth, 2002; Lustig, Rosenthal, Strausser y Haynes, 2000, Strausser y Lustig, 2003). De acuerdo con Godoy (1999) se le podría denominar como variable protectora o recurso de resistencia o competencia.

El sentido de coherencia refleja una posición básica ante la vida, una visión de mundo particular, una visión de vida ante acontecimientos inesperados y ante la vida misma (Antonowsky, 1987). De igual forma que las experiencias le pueden dar solidez al sentido de coherencia, también caracterizan la forma en que las personas las construyen. Esa forma de ver los hechos y de relacionarse con ellos se desarrolla durante los primeros 10 años de vida y se consolidan, a más tardar, a los 30 años. Después, el sentido de coherencia permanece hasta el fin de la vida sin cambios importantes. Se trata de una orientación disposicional en relación con los acontecimientos, las personas y las circunstancias de la vida, y en la que adopta una función de directriz (Antonowsky). El sentido de coherencia es una orientación general que se manifiesta como un sentimiento dinámico de confianza, de manera que los estímulos internos o externos son estructurados, predecibles y explicables. Por otro lado, se poseen los recursos para hacerle frente a los retos que se presenten y, finalmente, los estímulos son vistos y valorados como algo que vale la pena enfrentar y superar. Un sentido de coherencia fuertemente consolidado es base fundamental para un patrón de reacciones y acciones flexible y adaptativo (Antonowsky).

Antonowsky (1997) se refiere a tres factores fundamentales relacionados con el sentido de coherencia: 1) La comprensibilidad (componente cognoscitivo), se refiere a la medida en que una persona ordena, predice y explica estímulos internos y externos. Es decir, se trata de un aspecto cognitivo relacionado con el patrón de procesamiento de información. Con base en Moreno, González y Garrosa (1999) altos puntajes en este factor se asocian con una mayor capacidad para interpretar los estímulos del medio de manera no amenazante, aspecto que juega un papel central en la vivencia del estrés y, consecuentemente, en la calidad de vida de las personas, 2) la manejabilidad (componente instrumental), describe la confianza instrumental de la persona, su convicción de 
poder superar las situaciones de la vida presente o futura, con las propias fuerzas o con ayuda de los demás y 3) la significatividad (componente motivacional), tiene que ver con el sentido de la propia vida y se le considera el aspecto motivacional emotivo del sentido de coherencia. Representa el grado de compromiso de la persona en diferentes áreas de la vida. Esta dimensión denota una inversión emocional en la vida y tiene que ver con la visión del sujeto de ciertas áreas de ésta como dignas de invertir tiempo y energía. Puntuaciones altas en esta dimensión son evidencia de que difícilmente la vida será vista como poco provechosa e insignificante, contribuyendo de manera importante al proyecto de vida de las personas.

De acuerdo con los planteamientos de Antonowsky (1997), el sentido de coherencia puede ejercer influencia directa sobre el cerebro, el sistema inmunológico y el sistema hormonal de las personas, desencadenando reacciones distintas en diferentes niveles, de manera que puede incidir en forma reguladora cuando presentan estados de tensión, o como filtro directo en el procesamiento de la información. Asimismo, el sentido de coherencia puede actuar de manera directa en el procesamiento del estrés, en la medida en que moviliza recursos que contribuyen con la reducción de la tensión (Lundquist, 1995; Johansson, Larsson y Hamrin, 1998; Poppius, Tenkanen, Kalimo y Heinsalmi, 1999). Finalmente, las personas que disponen de un sentido de coherencia fuerte, están en mejor condición de asumir conductas saludables, por ejemplo: tener una alimentación sana, tomar previsiones y evitar comportamientos no saludables.

Alrededor de esta problemática, en estudios realizados por Palsson, Hallberg, Norberg y Bjoervell (1996) se encontró una relación inversa entre los niveles de sentido de coherencia y la sensación de agotamiento crónico (burn out) producida por el trabajo. Es decir, cuanto más fuerte sea el sentido de coherencia, menor será la sensación de agotamiento emocional y despersonalización asociados con la vivencia de estrés crónico (Baker, North y Smith, 1997, Gilbar, 1998). En este mismo sentido, los trabajos reportados por Carmel, Anson, Levenson, Bonneh y Maoz (1991), Flannery y Flannery (1990), Moreno, Alonso y Alvarez (1997), Moreno González y Garrosa (1999) y Ryland y Greenfeld (1991) destacan el rol relevante que juega el sentido de coherencia como variable mediadora en los procesos de salud (Lustig, 2005).

Con base en la revisión anterior, se propuso analizar la calidad de vida, el sentido de coherencia y la satisfacción laboral de los(as) educadores(as) de los colegios técnicos de la Dirección Regional de Heredia.

\section{METODOLOGÍA}

Se trató de un estudio descriptivo y correlativo en el que se describe cómo es la calidad de vida de los(as) profesores(as) de colegios técnicos del Circuito de Heredia. Se midió una serie de dimensiones indicadoras de calidad de vida, y se relacionaron con el constructo sentido de coherencia y bienestar laboral.

\section{Sujetos}

Se contó con la participación de 25 educadores y 56 educadoras de los colegios técnicos del Circuito de Heredia. La edad promedio general fue de 39 años. El 43,2 \% trabaja en condición de interino(a) y el 55,6 \% en condición de propietario(a). En términos promedio, el grupo tiene un tiempo de 10,44 años de laborar para el Ministerio de Educación. 


\section{Instrumentos}

La información requerida en este estudio se recopiló con base en el Cuestionario de salud (SF-36) (Ware y Sherbourne, 1992), las escalas de Sentido de coherencia (Antonowsky, 1987) y de Bienestar laboral.

\section{El cuestionario de salud (SF-36)}

Se trata de una escala genérica que proporciona un perfil de la Calidad de vida relacionada con la salud (CVRS), la cual es aplicable tanto a la población en general como a pacientes (Vilagut, Ferrer, Rajmil y Rebollo, 2005). El cuestionario está compuesto por 36 ítemes que valoran tanto los estados positivos como los negativos de la salud. Los 36 ítemes abarcan las siguientes escalas:

- Función física, compuesto por los ítemes 3, 4, 5, 6, 7, 8, 9, 10, 11 y 12. Esta dimensión se refiere al grado en el que la salud limita las actividades físicas tales como el autocuidado, caminar, subir escaleras, inclinarse, coger y llevar cargas o pesos, y los esfuerzos moderados e intensos.

- Rol físico, integrado por los ítemes 13, 14, 15 y16. Se trata del grado en el que la salud física interfiere con el trabajo y en otras actividades diarias, incluyendo un rendimiento menor que el deseado, la limitación en el tipo de actividades realizadas o la dificultad en la realización de actividades.

- Dolor corporal, conformada por los ítemes 21 y 22. Tiene que ver con la intensidad del dolor y su efecto en el trabajo habitual, tanto fuera de la casa como dentro de ella.

- $\quad$ Salud general, integrada por los ítemes 1, 33, 34, 35 y 36. Valoración personal de la salud que incluye la salud actual, las perspectivas de salud en el futuro y la resistencia a enfermar.

- Vitalidad, compuesta por los ítemes 23, 27, 29 y 31. Se refiere al sentimiento de energía y vitalidad, frente al sentimiento de cansancio y agotamiento.

- Función social, incluye los ítemes 32 y 20. Grado en el que los problemas de salud física o emocional interfieren en la vida social habitual.

- Rol emocional, ítemes 17, 18 y 19. Grado en el que los problemas emocionales interfieren en el trabajo u otras actividades diarias, incluyendo reducción en el tiempo dedicado a esas actividades, rendimiento menor que el deseado y disminución del esmero en el trabajo.

- $\quad$ Salud mental, ítemes 24, 25, 26, 28 y 30. Salud mental general, incluyendo depresión, ansiedad, control de la conducta y bienestar general.

Cada ítem se puntúa de 0 a 100, en el que 0 es el peor estado de salud y 100 el mejor. El puntaje correspondiente a cada dimensión se suma luego de la conversión y se divide entre el número de ítemes para obtener el promedio.

El instrumento incluye un ítem de transición que pregunta por el cambio en el estado de salud general respecto al año anterior; sin embargo, ese ítem no se utiliza para el cálculo de ninguna de las escalas, pero proporciona información valiosa sobre el cambio percibido en el estado de salud. Se reporta una consistencia interna superior a 0,7 (alfa de Cronbach) en la mayoría de las dimensiones (rango de 0,71-0,94), excepto en la dimensión de relación social (alfa =0,45). Los coeficientes de correlación intraclase, oscilan entre 0,58 y 0,99 (Alonso, Prieto y Antó, 1995). 


\section{Escala Sentido de coherencia (ver cuál solución factorial se utilizará)}

Instrumento constituido por 29 ítemes agrupados en tres subescalas que evalúan cada una de la tres dimensiones del constructo "Sentido de Coherencia": 1) comprensibilidad. Describe la medida en que una persona ordena, predice y explica estímulos internos y externos. Se trata de un aspecto cognitivo relacionado con el patrón de procesamiento de información. Tiene 11 ítemes, 2) manejabilidad. Describe la confianza instrumental de la persona, su convicción de poder superar las situaciones de la vida presente o futura con las propias fuerzas o con ayuda de los demás. Antonowsky (1997) define esa convicción como un patrón de procesamiento de información cognitivo-emocional). Tiene 10 ítemes y 3) significatividad. Se refiere al sentido de la propia vida y se le considera el aspecto emotivo-motivacional del Sentido de coherencia (Antonowsky, 1997). Tiene 8 ítemes. La escala de respuestas presenta 7 categorías (de 1 a 7) que forman un continuo bipolar limitado en los extremos por dos frases de referencia a partir de las cuales se debe situar el sujeto. Las puntuaciones expresan la fortaleza del Sentido de coherencia, ésta es mayor cuanto mayor sea la puntuación obtenida.

La versión utilizada es la adaptada por Moreno, Alonso, y Álvarez (1997). En cuanto a la consistencia interna de la escala se reporta un alfa de Conbrach con un rango que oscila entre 0,82-0,95. En cuanto a la estabilidad temporal se han encontrado correlaciones que van desde 0,54 después de 2 años, hasta 0,97 luego de 5 años. La validez de constructo, en su modalidad convergente, se ha obtenido por medio de la correlación de la escala con instrumentos análogos como: la escala de Hardiness de Kobasa $(0,50)$, el Inventario de estresores de Sheridan $(0,56)$ y con la escala de Potencial adaptativo de Colby $(0,75)$.

\section{Escala de Satisfacción laboral}

Esta escala se desarrolló con base en el trabajo realizado por Meliá, Pradilla, Martí, Sancerni, Oliver y Tomás (1986; 1989; 1990). Este instrumento responde, exclusivamente, a los propósitos particulares del estudio y se basa en la idea de que la vivencia personal subjetiva de las condiciones que caracterizan el contexto del trabajo, determinan el grado de satisfacción laboral. Está conformado por 15 ítemes relacionados con condiciones que caracterizan el contexto laboral del docente en el nivel de secundaria. Incluye aspectos relacionados con la infraestructura, ambiente físico, reglas de trabajo, relaciones interpersonales, remuneración económica, reconocimiento, entre otros aspectos. Sobre la base de una escala de 5 puntos, en la que $1=$ insatisfecho, $2=$ poco satisfecho, $3=$ medianamente satisfecho, 4 = satisfecho y $5=$ bastante satisfecho, se pregunta por el nivel de satisfacción, en relación con cada una de las condiciones mencionadas.

\section{PROCEDIMIENTOS}

Se confeccionó un cuadernillo con los instrumentos utilizados en el estudio y luego se les solicitó a los directores y las directoras de los colegios involucrados en el estudio, distribuir el material a la mayor cantidad posible de docentes, en sus respectivos colegios. Los cuestionarios fueron distribuidos durante el mes de abril de 2008, y se hizo énfasis en la necesidad de que cada uno de ellos fuera contestado de manera completa. Se concedió un tiempo de 15 días para que las personas devolvieran los cuestionarios. Finalizado el período de espera, se procedió con la construcción de la base de datos, para finalmente, concluir con el análisis de los datos. 


\section{Análisis estadísticos}

Los análisis de los datos recolectados se realizaron con base en el paquete estadístico para las ciencias sociales SPSS, versión 15. Se trabajó con estadísticas descriptivas, correlativas e inferenciales. En las pruebas de contraste de hipótesis se utilizó un nivel de significancia igual o menor a 0.05.

\section{RESULTADOS}

\section{Bienestar laboral}

Se realizó un análisis factorial exploratorio a partir de los 15 ítemes correspondientes a la valoración de Bienestar laboral. Se utilizó como método de extracción el análisis de componentes principales y la normalización varimax con Kaiser como método de rotación. Después de varios análisis, se escogió la solución de tres factores, los cuales explicaron un 52,01 \% de la varianza.

Tabla $\mathrm{N}^{\mathrm{o}} 1$

Solución de tres factores y respectivos pesos factoriales

\begin{tabular}{|rl|c|c|c|}
\hline & \multicolumn{1}{|c|}{ Ítemes } & Factor 1 & Factor 2 & Factor 3 \\
\hline 3. & El ambiente social & 0,78 & & \\
4. & Las reglas de trabajo & 0,80 & & \\
5. & El reconocimiento de mi trabajo & 0,61 & & \\
6. & La relación con los(as) compañeros(as) & 0,66 & & \\
8. & La relación con el (la) jefe (a) & 0,70 & 0,63 & \\
& & & 0,57 & \\
1. & Condiciones de infraestructura & & 0,61 & \\
2. & Condiciones ambientales & & 0,44 & \\
11. & Las posibilidades de capacitación & & 0,66 & \\
12. & La remuneración económica & & 0,52 & 0,63 \\
13. & Las posibilidades de ascenso & & & 0,59 \\
15. & La cantidad de horas que labora & & & 0,66 \\
& & & & 0,60 \\
7. & El tipo de trabajo & & & \\
9. & La cantidad de trabajo & & & \\
10. & La relación con los(as) estudiantes & & & \\
14. & La ubicación del colegio & & & \\
\hline & & & & \\
\hline
\end{tabular}

Nota. Resultado del análisis factorial exploratorio de los ítemes sobre la valoración de Bienestar laboral. 
De acuerdo con el análisis factorial realizado, el primer factor reúne ítemes que están relacionados con el ambiente social que se vive en la Institución y con el reconocimiento que tienen las personas en su lugar de trabajo, por lo que se le denomina ambiente social y reconocimiento. El segundo factor aglomera ítemes vinculados con aspectos propios de la infraestructura de la Institución, con las posibilidades de ascenso en ella y con la remuneración que reciben por el trabajo. A este factor se le denomina condiciones de infraestructura, ascenso y remuneración. Finalmente, el tercer factor agrupa condiciones relacionadas con la cantidad de trabajo que se realiza, el acceso a la Institución y las relaciones con los estudiantes. Este factor se denomina cantidad y tipo de trabajo, acceso a la Institución y relaciones con estudiantes.

Análisis diferenciados de los puntajes promedio registrados en cada uno de los factores correspondientes con Bienestar laboral, evidencian que de acuerdo con la escala de valoración de los ítemes de esta escala, tanto en el factor ambiente social y reconocimiento, como en el factor cantidad de trabajo, tipo y relaciones con estudiantes, los(as) encuestados(as) manifiestan, en términos promedio, estar satisfechos con las condiciones laborales reflejadas en ambos factores. Sin embargo, en el factor condiciones infraestructurales, acceso y remuneración, el valor promedio registrado evidencia niveles de satisfacción calificados como intermedios.

Tabla $\mathrm{N}^{\mathrm{o}} 2$

Puntajes promedio y desviaciones típicas en los factores del Bienestar laboral

\begin{tabular}{|l|c|c|}
\multicolumn{1}{|c|}{ Factores } & Promedio & Desviación típica \\
\hline F1 Ambiente social y reconocimiento & 4,07 & 0,65 \\
\hline F2 Condiciones infraestructurales, ascenso y remuneración & 3,39 & 1,05 \\
\hline F3 Cantidad de trabajo, tipo y relaciones con estudiantes & 4,29 & 0,56 \\
\hline
\end{tabular}

Nota. Resultado del análisis factorial exploratorio de los ítemes sobre la valoración de Bienestar laboral.

De manera específica, la distribución de frecuencias correspondientes a cada uno de los factores mostró, para el caso del factor ambiente social y reconocimiento que: el 29,6 \% de los encuestados expresó niveles medios de satisfacción; el 70,4 \% manifestó sentirse satisfecho con las condiciones ambientales y el reconocimiento que reciben en cada una de las instituciones donde laboran. En el caso del factor condiciones infraestructurales, ascenso y remuneración, el 75,3\% se mostró medianamente satisfecho. Sólo un 24,7 \% dijo estar satisfecho. Finalmente, el 76,5 \% afirmó estar satisfecho con cantidad de trabajo, tipo y las relaciones con los estudiantes. Un 23,5 $\%$ manifestó estar medianamente satisfecho.

\section{Calidad de vida}

En cuanto a la valoración de la salud actual comparada con la salud en el pasado reciente (un año atrás), el 12, $3 \%$ dijo sentirse peor, el 43,2 \% más o menos igual, el 18, $3 \%$ algo mejor y el 23, $5 \%$ mucho mejor. 
Tabla $\mathrm{N}^{\circ} 3$

Valores promedio para los factores del SF-36

\begin{tabular}{|l|c|c|c|}
\hline \multicolumn{1}{|c|}{ Factores } & Promedio & Desviación típica & Valor máximo \\
\hline Función física & 84,93 & 19,64 & 100 \\
Rol físico & 83,95 & 32,42 & 100 \\
Función social & 77,31 & 26,07 & 100 \\
Salud mental & 75,85 & 18,29 & 100 \\
Rol emocional & 75,30 & 40,74 & 100 \\
Salud general & 72,22 & 20,17 & 100 \\
Dolor corporal & 71,94 & 29,14 & 100 \\
Vitalidad & 62,65 & 21,30 & 100 \\
\hline
\end{tabular}

Nota. Resultado del análisis de los ítemes sobre la valoración de Calidad de vida.

Los datos diferenciados para cada factor correspondiente al SF-36 mostraron que los mayores puntajes se relacionan con los factores función física y rol físico. El menor puntaje se nota en el factor vitalidad. Las puntuaciones promedio correspondientes a los factores dolor corporal, salud general, función social, rol emocional y salud mental oscilan entre 71,94 y 77,31. En lo global, de un máximo posible de 800 puntos, se obtuvo 604,15, lo que representa un 75,51\%.

Con el propósito de identificar la cantidad de personas que obtenían puntajes con calificación excelente en los factores del SF-36, se calculó la distribución de frecuencias para cada uno de los factores supra-mencionados (véase Tabla $N^{\circ}$ 4). Los resultados muestran que los factores en los que menos cantidad de personas califica como excelente su salud son: vitalidad, salud general y salud mental. Asimismo, los factores en los que la mayor cantidad de personas se califican como excelente son: rol físico y rol emocional. En el resto de los factores las porcentajes oscilan entre el 28,4\% para el factor función física y el $42 \%$ para el factor función social.

\section{Tabla $\mathrm{N}^{\mathrm{o}} 4$}

Porcentaje de encuestados con calificación excelente en los factores del SF-36

\begin{tabular}{|l|c|}
\hline \multicolumn{1}{|c|}{ Dimensiones de la calidad de vida } & Calificación excelente \\
\hline Función física & $\mathbf{2 8 , 4 \%}$ \\
Rol físico & $77,8 \%$ \\
Dolor corporal & $34,6 \%$ \\
Salud general & $8,6 \%$ \\
Vitalidad & $4,9 \%$ \\
Función social & $\mathbf{4 2 , 0 \%}$ \\
Rol emocional & $69,1 \%$ \\
Salud mental & $12,3 \%$ \\
\hline
\end{tabular}

Nota. Resultado del análisis de los ítemes sobre la valoración de Calidad de vida. 


\section{Sentido de coherencia}

Los puntajes registrados en cuanto al sentido de coherencia muestran para el factor vida, un logro del 77,82 \% respecto del puntaje máximo posible. Asimismo, para el factor sentimientos, el porcentaje alcanzado fue de un 71,78 \%. Finalmente, en cuanto a coherencia global, se alcanzó un $72,07 \%$.

Tabla $\mathrm{N}^{\mathrm{o}} 5$

Promedio y desviación típica para las dimensiones del Sentido de coherencia

\begin{tabular}{|l|c|c|c|}
\hline \multicolumn{1}{|c|}{ Factores } & Promedio & Desviación típica & Puntaje máximo \\
\hline F1 Vida (significancia) & 70,82 & 12,48 & 91 \\
F2 Sentimientos (manejabilidad /comprensibilidad) & 40,20 & 18,34 & 56 \\
\hline Coherencia global & $\mathbf{1 1 1 , 0 3}$ & $\mathbf{1 8 , 3 4}$ & $\mathbf{1 5 4}$ \\
\hline
\end{tabular}

Nota. Resultado del análisis de los ítemes sobre la valoración del Sentido de coherencia.

El análisis de las relaciones entre los distintos factores del SF-36, del Sentido de coherencia y de la escala de Bienestar laboral (véase Tabla $\mathrm{N}^{\mathrm{o}}$ 6) muestra que, con excepción de los factores rol emocional y función física, así como rol emocional y rol físico, y salud mental y rol físico, todas los factores restantes del SF-36 se correlacionan, de manera significativa, entre sí. Asimismo, el factor vida de la escala de Sentido de coherencia se correlaciona de manera significativa y positiva, con el factor sentimientos de la misma escala. También el factor vida se correlaciona, significativamente, con los factores salud general, función social, rol emocional y salud mental. Por otro lado, se observan relaciones importantes entre el factor sentimientos y los factores salud general, vitalidad, función social, rol emocional y salud mental. En cuanto a los factores de bienestar laboral, todos se correlacionan significativa y positivamente entre sí. De manera particular, el factor ambiente social y reconocimiento de Bienestar laboral, se correlacionó significativamente con los factores función física, rol físico, salud general; asimismo con los factores vida y sentimientos de la escala Sentido de coherencia. El factor condiciones infraestructurales, ascenso y remuneración se asoció de manera significativa con los factores función física y vitalidad del SF-36; también se relacionó significativamente con el factor vida. Asimismo, el factor cantidad y tipo de trabajo, acceso a la institución y relaciones con estudiantes, sólo se correlacionó significativamente con el factor vitalidad del SF-36. 
Tabla $\mathrm{N}^{\mathrm{o}} 6$

Matriz de correlaciones para los factores del SF-36, sentido de coherencia bienestar laboral

\begin{tabular}{|c|c|c|c|c|c|c|c|c|c|c|c|c|c|}
\hline & $\mathbf{a}$ & b & c & d & e & $\mathbf{f}$ & g & h & $\mathbf{i}$ & $\mathbf{j}$ & $\mathbf{k}$ & $\mathbf{m}$ & $\mathbf{n}$ \\
\hline \multicolumn{14}{|l|}{$\begin{array}{l}\text { FF } \\
\text { (a) }\end{array}$} \\
\hline $\begin{array}{l}\mathbf{R F} \\
\text { (b) }\end{array}$ & $.63 * *$ & & & & & & & & & & & & \\
\hline $\begin{array}{l}\text { DC } \\
\text { (C) }\end{array}$ & $.49 * *$ & $.65^{* * *}$ & & & & & & & & & & & \\
\hline $\begin{array}{l}\text { SG } \\
\text { (d) }\end{array}$ & $.58 * *$ & $.41 * *$ & $.44 * *$ & & & & & & & & & & \\
\hline $\begin{array}{l}\text { VIT } \\
\text { (e) }\end{array}$ & $.45^{* * *}$ & $.37 * *$ & $.58 * *$ & $.48 * *$ & & & & & & & & & \\
\hline $\begin{array}{l}\text { FS } \\
\text { (f) }\end{array}$ & $.46^{* * *}$ & $.47 * *$ & $.45 * *$ & $.60 * *$ & $.55^{* * *}$ & & & & & & & & \\
\hline $\begin{array}{l}\text { RE } \\
(\mathrm{g})\end{array}$ & .15 & .17 & $.24 *$ & $.29 *$ & $.47 * *$ & $.65^{* * *}$ & & & & & & & \\
\hline $\begin{array}{l}\text { SM } \\
\text { (h) }\end{array}$ & $.37 * *$ & .21 & $.42 * *$ & $.53 * *$ & $.71 * *$ & $.60 * *$ & $.61 * *$ & & & & & & \\
\hline $\begin{array}{l}\text { VID } \\
\text { (i) }\end{array}$ & .02 & .02 & .01 & $.30 * *$ & .15 & $.28 * *$ & $.27 *$ & $.39 * *$ & & & & & \\
\hline $\begin{array}{l}\text { SEN } \\
(\mathrm{j})\end{array}$ & .13 & .04 & .15 & $.34 * *$ & $.24 *$ & $.46^{* * *}$ & $.51 * *$ & $.47 * *$ & $.49 * *$ & & & & \\
\hline $\begin{array}{l}\text { B1 } \\
\text { (k) }\end{array}$ & $.26^{*}$ & $.24 *$ & .18 & $.29 * *$ & .15 & .20 & .15 & .21 & $.33^{* *}$ & $.22 *$ & & & \\
\hline $\begin{array}{l}\text { B2 } \\
\text { (m) }\end{array}$ & $.24 *$ & .15 & .19 & .20 & $.28^{*}$ & .04 & .04 & .17 & $.30 * *$ & .14 & $.44 * *$ & & \\
\hline $\begin{array}{l}\text { B3 } \\
\text { (n) }\end{array}$ & .18 & .10 & .10 & .20 & $.28^{*}$ & .07 & .12 & .21 & .19 & .11 & $.45 * *$ & $.50 * *$ & \\
\hline
\end{tabular}

$* \mathrm{p}<0.05$

$* * \mathrm{p}<0.01$

Nota . FF = función física, $\mathrm{RF}=$ rol físico, $\mathrm{DC}=$ dolor corporal, $\mathrm{SG}=$ salud general, $\mathrm{VIT}=$ vitalidad, $\mathrm{FS}=$ función social, $\mathrm{RE}=$ rol emocional, $\mathrm{SM}=$ salud mental, $\mathrm{VID}=$ vida, $\mathrm{SEN}=$ sentimientos, $\mathrm{B} 1=$ ambiente social y reconocimiento, B2 = condiciones de infraestructura, ascenso y remuneración y B3 = cantidad y tipo de trabajo, acceso a la institución y relaciones con estudiantes.

En cuanto al análisis de efectos simples, se encontraron datos significativos sólo para el caso de la comparación entre las personas de mayor experiencia laboral y las de menor experiencia. Para tales efectos se conformaron tres grupos, a partir de la experiencia laboral. El grupo de menos experiencia laboral (1-5 años), el grupo de experiencia laboral intermedia (6-12 años) y el grupo de mayor experiencia (13-27 años). De manera particular, las personas de menor experiencia mostraron un puntaje significativamente mayor que los de mayor experiencia en el factor función física del SF-36 ( F = 4,20; p < 0.05). La prueba post hoc de Scheffé mostró que el grupo de menor 
experiencia laboral $(X=93,51)$, obtuvo puntajes muy superiores a los que alcanzó el grupo con experiencia intermedia $(X=80,0)$ y el de mayor experiencia $(X=81,29)(p<0.05)$. Sin embargo, entre el grupo con experiencia intermedia y el de mayor experiencia no hubo diferencias significativas $(\mathrm{p}>0.05)$.

También, en cuanto a la condición laboral, los interinos comparados con los propietarios, mostraron una puntuación más alta en relación con el factor función física. El puntaje promedio de los interinos fue de 91,42 y el de los propietarios de 81,0 ( $\mathrm{t}=2,52 ; \mathrm{p}<0.05)$. En relación con el resto de los factores, tanto del SF-36, como del Sentido de coherencia y del Bienestar laboral no hubo diferencias importantes ( $\mathrm{p}>0.05)$.

Finalmente, se analizó el efecto de las interacciones entre las variables: sexo-edad, sexo-experiencia, sexo-condición laboral, experiencia-edad y experiencia-condición laboral, sobre las dimensiones del Cuestionario SF-36, Sentido de coherencia y Bienestar laboral. Los análisis univariados de varianza no reportaron interacciones significativas en ninguno de los casos $(p>0.05)$.

\section{DISCUSIÓN}

El propósito de este estudio fue analizar la Calidad de vida, el Sentido de coherencia y el Bienestar laboral de los(as) profesores(as) de los colegios técnicos ubicados en la Dirección regional de Heredia.

Los resultados correspondientes al Bienestar laboral mostraron que los(as) educadores(as) encuestados(as) se sienten, en su mayoría, satisfechos con las condiciones laborales prevalecientes en sus instituciones, lo que podría interpretarse como un buen nivel. De acuerdo con Duro (2005) esto podría considerarse como el reflejo de un buen nivel de logro de esperanzas y de aspiraciones individuales. Sin embargo, existe un porcentaje importante de educadores(as) que manifiesta no sentirse conforme con las circunstancias propias de sus centros de trabajo; en consecuencia, su nivel de Bienestar laboral no es el mejor. Especialmente crítico es el nivel de Bienestar laboral, en relación con las condiciones infraestructurales de los centros de trabajo, las posibilidades de ascender que existen en las instituciones y el pago que reciben por el ejercicio de la profesión. En el fondo, se trata de condiciones que escapan de las posibilidades que manejan los administradores educativos y que más bien responden a condiciones económicas de orden nacional, o bien, a políticas particulares que no priorizan en la promoción de la calidad de vida del educador, lo que podría atentar contra el Bienestar laboral y, consecuentemente, reducir la identificación y el compromiso del educador con su quehacer.

La Calidad de vida de los(as) encuestados(as) se muestra, relativamente, pobre, en el factor vitalidad. Esto refleja la sensación de falta de energía en el(la) educador(a) o, bien, el peso que tiene la sensación de cansancio y de agotamiento en estos(as) trabajadores(as). Pareciera evidente que el quehacer del(a) educador(a) es una tarea que se asocia con altos grados de fatiga, lo que puede atentar contra su salud. A pesar de ello, el grupo encuestado, en su generalidad, no muestra limitaciones importantes para realizar su trabajo a causa de problemas de salud relacionados con su bienestar físico. Al vincular el tema de la capacidad de función física y rol físico con el componente vitalidad, se puede afirmar que aunque no haya restricciones orgánicas importantes para desempeñar la función educadora, sí existe un sentimiento importante de falta de energía y de vitalidad, lo que permite suponer reducciones importantes en la capacidad y calidad del trabajo que desarrolla el(la) educador(a). Muy relacionado con las observaciones anteriores, los resultados encontrados evidencian un peso importante de la presencia de dolor corporal como acompañante 
en el desempeño del trabajo de los(as) trabajadoras(es) encuestados(as), así como, también, una valoración personal relativamente deteriorada de su salud actual y de su resistencia a enfermarse en un futuro inmediato. A pesar de todo ello, las personas encuestadas reflejan una vida social dinámica, aun cuando existan problemas físicos y/o emocionales relacionados con su quehacer laboral, y muestran una valoración aceptable aunque no la mejor de su bienestar general.

En cuanto al Sentido de coherencia general, los(as) educadores(as) encuestados(as) manifiestan poseer una autovaloración de su competencia psicológica aceptable, aunque no óptima. Esto puede afectar la salud general de estos(as) educadores(as) y su calidad de vida (Antonovsky, 1987; Ebert, Tucker y Roth, 2002; Strausser y Lustig, 2003). El sentimiento de confianza de los(as) educadores(as) encuestados(as) no parece ser el mejor, lo que, de alguna manera, se podría asociar con patrones conductuales poco adaptativos en su comportamiento (Antonovsky, 1997). Éstas son situaciones que atentan no sólo contra la calidad de vida del educador, sino, también, contra un desempeño docente de calidad. De manera particular, en el factor denominado vida, las personas encuestadas reflejan patrones de relación cognoscitivos aceptables (optimistas) con los contextos inmediato y mediato, aunque también evidencian la presencia de actitudes negativas ante la vida, lo que, además, puede vincularse con estilos cognitivos pesimistas en cuanto al ordenamiento, predicción y explicación de los estímulos internos y externos a los que están sujetos.

Un comportamiento similar se observa en el caso del factor sentimientos. Al respecto, los resultados encontrados reflejan un nivel aceptable de convicción de control sobre las situaciones y acontecimientos, así como de confianza en la relación con otros. A pesar de ello, también se deja ver cierto grado de incertidumbre asociado a las circunstancias internas y externas propias de cada educador(a); asimismo, cierto nivel de desconfianza en relación con las posibilidades que se tienen para superar las situaciones de la vida presente y futura.

Los resultados, igualmente, indican que el sentido propio de la vida, aspecto que se relaciona estrechamente con las actitudes ante la existencia, y que interviene de manera decisiva en la dimensión emotiva y motivacional del sentido de coherencia, se vincula de manera importante con la valoración actual del estado de salud general y con las perspectivas futuras de salud. Esto significa que todo esfuerzo que se haga en procura del desarrollo y de la consolidación de las actitudes positivas ante la vida, ya sea en el contexto laboral o en general, no sólo fortalece el Sentido de coherencia, sino que, también, genera la sensación de un buen estado de salud, percepción subjetiva fundamental para una buena calidad de vida. Estrechamente relacionado con lo anterior, también se reduciría la probabilidad de que los problemas tanto físicos como emocionales afecten el desempeño cotidiano y la salud, así como la sensación de bienestar general. Por otro lado, cuanto mayor es el sentimiento de control y la sensación de confianza en las relaciones con otros, mayor es, también, la valoración que se hace del estado de salud actual, del sentimiento de energía y de vitalidad. Asimismo, se reduce la interferencia de los problemas de salud física y emocional en el desarrollo de una vida social activa y se tendrá una mayor sensación de bienestar general.

Un buen estado de Bienestar laboral, en lo relacionado con el ambiente social y el reconocimiento, se vincula, de manera importante, con la valoración subjetiva de una capacidad física aceptable para desempeñar actividades cotidianas, con la sensación general de buena salud, con la presencia de actitudes positivas ante la vida y con la convicción de control sobre los acontecimientos y la confianza en la relación con otros. Asimismo, el Bienestar laboral, en lo tocante a condiciones infraestructurales, ascenso y remuneración, se relacionó, significativamente, con la capacidad para desempeñar tareas cotidianas desde el punto de vista físico, con el sentimiento de energía y de vitalidad y con la convicción de control y de confianza en la relación con otros. También se encontró, que el Bienestar laboral, asociado con la cantidad y calidad del trabajo que se realiza y 
con las relaciones con estudiantes, se vincula, de manera importante, con la sensación de energía y de vitalidad.

Entre los factores utilizados para contrastar hipótesis, sólo la experiencia y la condición laboral arrojaron datos significativos. En el primero de los casos, las personas con menos tiempo de trabajar como educadores (as), comparadas con las que tienen más tiempo, hacen una mejor valoración subjetiva de su capacidad física para el desempeño de las tareas cotidianas. Esto significa que el tiempo de trabajo como educador(a) y las consecuencias asociadas al ejercicio profesional, afectan negativamente la valoración subjetiva que se hace de las posibilidades de desempeño físico. Igual comportamiento se registró para el caso de los interinos y los propietarios. Los interinos, comparados con los propietarios, a pesar de su condición de inestabilidad laboral, muestran una mejor valoración subjetiva de su estado y su capacidad física para hacerle frente a su desempeño cotidiano. Es importante señalar que la condición de propietario, usualmente, se asocia con un tiempo prolongado de laborar, lo que refuerza la idea de que el tiempo de trabajo como educador(a) incide negativamente en el estimado que se haga de la capacidad funcional para enfrentar la cotidianidad.

Como conclusión general puede afirmarse que aunque la calidad de vida del(a) educador(a) sea, en término promedio, aceptable, hay mucho que invertir en programas que fortalezcan la calidad de vida de estos(as) profesionales, máxime la importancia que se supone tiene la educación en el desarrollo social y humano de los pueblos. Asimismo, es preciso pensar en propuestas y acciones concretas que potencien el desarrollo de actitudes positivas y de autoconfianza, específicamente, en los(as) educadores(as) de los colegios técnicos y, en general, de las demás instituciones educativas. Probablemente, al promocionar una mejor calidad de vida y un buen sentido de coherencia, se tendrá como resultado un mejor nivel de satisfacción laboral y, como consecuencia, un mayor compromiso y disfrute del ejercicio profesional del(a) educador(a).

\section{REFERENCIAS}

Alonso, J., Prieto, L. \& Antó, J. M. (1995). La versión española del SF-36 Health Survey (Cuestionario de Salud SF-36): un instrumento para la medida de los resultados clínicos. Medicina Clínica, 104 (20), 771-776.

Antonowsky, A. (1987). Unraveling the Mystery of Health: How People Manage Stress and Stay Well. San Francisco, United States: Jossey-Bass.

Antonowsky,A.(1997). Salutogenese.Zur Entmystifizierung der Gesundheit. Tübingen, Deutschland: Dgvt.

Baker, M., North, D. \& Smith, D. F. (1997). Burnout, sense of coherence and sources of salutogenesis in social workers. Psychology: A Journal of Human Behavior, 34, 22-26.

Bravo, M., Peiró, J. M. \& Rodríguez, I. (1996). Satisfacción laboral. En J. M. Peiró y F. Prieto (Eds.). Tratado de psicología del trabajo. Vol. 2, 343-397.

Brengelmann, J. C. (1986). Stress, superación y calidad de vida en personas sanas y enfermas. Evaluación Psicológica, 2, 47-77. 
Carmel, S., Anson, O., Levenson, A., Bonneh, D. \& Mahoz, B. (1991). Life events, sense of coherence and health: gender differences on the kibbutz. Social Science and Medicine, 32, 1089-1096.

Diener, E. (1994). Assessing subjective well-being: Progress and opportunities. Social Indicators Research, 31, 103-157.

Duro, A. (2005). Calidad de vida laboral y psicología social de la salud en el trabajo: hacia un modelo de compoentes comunes para explicar el bienestar laboral psicológico y la salud mental laboral de origen psicosocial. Revista del Ministerio de Trabajo y Asuntos Sociales. Universidad Rey Juan Carlos. España.

Ebert, S., Tucker, D. \& Roth, D. (2002). Psychological resistence factors as predictors of general health status and physical symptom reporting. Health and Medicine, 7, 363-375.

Flannery, R. B. \& Flannery, G. J. (1990). Sense of coherence, life stress, and psychological distress: a prospective methodological inquiry. Journal of Clinical Psychology, 46, 415-420.

Gilbar, O. (1998). Relationship between burnout and sense of coherente in health social workers. Social Work in Health Care, 26, 39-49.

Godoy, J. F. (1999). Psicología de la salud: delimitación conceptual. En M.A. Simón (Ed.), Manual de Psicología de la Salud. Fundamentos, metodología y aplicaciones (pp. 39-70). Madrid, España: Biblioteca Nueva.

Johansson, I., Larsson, G. \& Hamrin, E. (1998). Sense of Coherence, Quality of Life, a Function among Elderly Hip Fracture. Aging, 10, 377-384.

Levy, L. \& Anderson, L. (1980). La tensión psicosocial. Población, ambiente y calidad de vida. México: El Manual Moderno.

Lundquist, T. (1995). Chronic Cannabis Use and the Sense of Coherence. Life Science, 56, 21452150 .

Lustig, D. (2005). The Adjustment Process for Individuals with Spinals Cord Injury: The effect of perceived premorbid sense of coherence. Rehabilitation Counseling Bulletin, 48 (3), 146156.

Lustig, D., Rosenthal, D., Strausser, D. \& Haynes, K. (2000). The Relationship of Sense of Coherence to Life Satisfaction for Students with Disabilities. Rehabilitation Counseling Bulletin, 43, 134-141.

Meliá, J. L., Peiró, J. M. \& Calatayud, C. (1986). El cuestionario general de satisfacción en organizaciones laborales: Estudios factorials, fiabilidad y validez. Revista de Filosofía, Psicología y Ciencias de la Educación, 11 (3/4), 43-78. 
Meliá, J. L. \& Peiró, J. M. (1989). La medida de la satisfacción laboral en contextos organizacionales: El cuestionario de Satisfacción S 20/ 23. Psicologemas, 5, 59-74.

Meliá, J. L., Pradilla, J.F., Martí, N., Sancerni, M. D., Oliver, A. \& Tomás, J. M. (1990). Estructura factorial, fiabilidad y validez del cuestionario de satisfacción $S$ 21/26. Un instrumento con formato dicotómico orientado al trabajo profesional. Revista de Psicología Universitas Tarraconenses, 12 (1/2), 25-39.

Moreno, B., González, J. L. \& Garrosa, E. (1999). Burnout docente, sentido de coherencia y salud percibida. Revista de Psicopatología Clínica 4 (3), 163-180.

Moreno, B., Alonso, M. \& Álvarez, E. (1997). Sentido de coherencia, personalidad resistente, autoestima y salud. Revista de Psicología de la salud, 9, 115-138.

Nordenfelt, L. (1993). Quality of life, Health and Happiness. Aldershot, England: Avebury.

Palsson, M. B., Hallberg, I. R., Norberg, A. \& Bjoervell, H. (1996). Burnout, Empathy and Sense of Coherence among Swedish District Nurses before and After Systematic Clinical Supervision. Scandinavian Journal of Caring Sciences, 10, 19 -26.

Poppius, E., Tenkanen, L., Kalimo, R. \& Heinsalmi, P. (1999). The Sense of Coherence, Occupation and the Risk of Coronary Heart Disease in the Helsinski Heart Study. Social Science Medicine, 49, 109-120.

Requena, F. (2000). Satisfacción, bienestar y calidad de vida en el trabajo. Revista Española de Investigaciones Sociológicas (REIS), 92, 11-44.

Ryland, E. \& Greenfeld, S. (1991). Work, Stress and Well-being: An Investigation of Antonowskys Sense of Coherence Model. In P.L. Perrewe (Ed.), Handbook of Job Stress (special issue). Journal of Social Behavior and Personality, 6, 39-54.

Ryff, C. (1989). Happinessis is Everything, or Is It? Explorations on the Meaning of Psychological Well-being. Journal of Personality and Social Psychology, 57, 1069-1081.

Santos, R. (2000). Satisfacción, bienestar y calidad de vida en el trabajo. Revista española de investigaciones sociológicas, 92, 14-44.

Sánchez, F. (1996). La actividad física orientada hacia la salud. Madrid, España: Biblioteca Nueva.

Simón, M. A. (1999). Manual de Psicología de la salud. Fundamentos, metodología y aplicaciones. Madrid, España: Biblioteca Nueva.

Strausser, D. \& Lustig, D. (2003). The Moderating Effects of Sense of Coherence on Work Adjustment. Journal of Employment Counseling, 40, 129-140). 
Veenhoven, R. (1991). Questions on Happiness: Classical Topics, Modern Answers, Blind Spots. In F. Strack, M. Argyle \& N. Schwars (Eds.), Subjective Well-Being. An Interdisciplinary Perspective. Oxford, England: Pergamon Press.

Vilagut, G., Ferrer, M., Rajmil, L. \& Rebollo, P. (2005). El cuestionario de salud SF-36 español: una década de experiencia y nuevos desarrollos. Gaceta Sanitaria, Vol. 19, №. 2, 1-16.

Ware, J. E. \& Sherbourne C. D. (1992). The MOS 36 -Item Short Form Health Survey (SF-36). I. Med Care, 39, 473-483. 